\title{
Predicted Impact of Vaccination and Active Case Finding Measures to Control Epidemic of Coronavirus Disease 2019 in a Migrant-Populated Area in Thailand
}

\author{
Rapeepong Suphanchaimat $\mathbb{D}^{1,2}$ \\ Natthaprang Nittayasoot ${ }^{\prime}$ \\ Panithee Thammawijaya' \\ Pard Teekasap (D) ${ }^{3}$ \\ Kumnuan Ungchusak' \\ 'Division of Epidemiology, Department of \\ Disease Control, Ministry of Public \\ Health, Nonthaburi, I 1000, Thailand; \\ ${ }^{2}$ International Health Policy Programme, \\ Ministry of Public Health, Nonthaburi, \\ I I000, Thailand; ${ }^{3}$ Stamford International \\ University, Bangkok, I0II0, Thailand
}

Correspondence: Rapeepong

Suphanchaimat

Email rapeepong@ihpp.thaigov.ne
Background: Thailand experienced the first wave of Coronavirus Disease 2019 (COVID19) during March-May 2020 and has been facing the second wave since December 2020 The area facing the greatest impact was Samut Sakhon, a main migrant-receiving province in the country. The Department of Disease Control (DDC) of the Thai Ministry of Public Health $(\mathrm{MOPH})$ considered initiating a vaccination strategy in combination with active case finding (ACF) in the epidemic area. The DDC commissioned a research team to predict the impact of various vaccination and ACF policy scenarios in terms of case reduction and deaths averted, which is the objective of this study.

Methods: The design of this study was a secondary analysis of quantitative data. Most of the data were obtained from the DDC, MOPH. Deterministic system dynamics and compartmental models were exercised. A basic reproductive number $\left(\mathrm{R}_{0}\right)$ was estimated at 3 from the beginning. Vaccine efficacy against disease transmission was assumed to be $50 \%$. A total of 10,000 people were estimated as an initial population size.

Results: The findings showed that the greater the vaccination coverage, the smaller the size of incident and cumulative cases. Compared with a no-vaccination and no-ACF scenario, the $90 \%$-vaccination coverage combined with $90 \%$-ACF coverage contributed to a reduction of cumulative cases by $33 \%$. The case reduction benefit would be greater when $\mathrm{R}_{0}$ was smaller ( $\sim 53 \%$ and $\sim 51 \%$ when $\mathrm{R}_{0}$ equated 2 and 1.5 , respectively).

Conclusion: This study reaffirmed the idea that a combination of vaccination and ACF measures contributed to favourable results in reducing the number of COVID-19 cases and deaths, relative to the implementation of only a single measure. The greater the vaccination and ACF coverage, the greater the volume of cases saved. Though we demonstrated the benefit of vaccination strategies in this setting, actual implementation should consider many more policy angles, such as social acceptability, cost-effectiveness and operational feasibility. Further studies that address these topics based on empirical evidence are of great value.

Keywords: COVID-19, vaccine, active case finding, reproduction number, Thailand

\section{Introduction}

In late 2019, the world recognized Coronavirus Disease 2019 (COVID-19), a disease that is causing a rare global pandemic. ${ }^{1-3}$ As of 14 February 2021, more than 108 million COVID-19 cases were reported in 219 countries and the toll of infections increased at a speedy pace. ${ }^{4}$ The first wave of COVID-19 in Thailand was caused by clusters of local infections related to imported cases from other 
countries, local transmission in boxing stadiums, entertainment venues and other crowded public places. ${ }^{5}$ In response to this, the Thai Government introduced several measures to curb the outbreak; for instance, international travel restriction, fourteen-day quarantine for all international returnees, interprovincial travel prohibition, and social distancing. ${ }^{6}$

The Division of Epidemiology (DOE) under the Department of Disease Control (DDC) of the Ministry of Public Health (MOPH) has played a pivotal role in containing the outbreak. One of the key measures is active case finding (ACF) in communities.

However, Thailand is now facing a new challenge as the second wave of the epidemic emerged in late December 2020, and this time, the impact was more severe than the earlier wave. ${ }^{7}$ Before December 2020, the number of total cases nationwide was about 5000. The national figure skyrocketed after January 2021. As of 14 February 2021, the volume of cumulative nationwide cases amounted to 24,571 , almost triple the total cases reported in $2020 .^{8}$

The new wave of the epidemic was believed to originate from migrant workers in a large shrimp market in the inner city of Samut Sakhon, a vicinity province of Bangkok. The province is a home to more than 11,000 factories with approximately 400,000 migrant workers (comprising those holding legitimate work permit and those without). The majority of the workers are from Myanmar. The living conditions of these workers are quite crowded, making social distancing or using masks all the time difficult. ${ }^{9}$ A rapid survey in early January 2021 by the DOE found about one fifth of the factories had some degree of infected workers, varying from less than $5 \%$ to more than $20 \%$. The daily incident cases in Samut Sakhon numbered about 100-150 throughout January 2021..$^{8}$ On certain days when the ACF was conducted, the incidence cases exceeded 800. Migrant workers accounted for approximately $80 \%$ of the total cases and most were identified by mass COVID-19 testing in migrant communities (as part of the ACF). City lockdowns and strict social distancing campaigns were also implemented. $^{10}$ Although numerous measures were enforced, the case toll still seemed to grow; and, at the time of writing, there was no sign that the case had reached its acme.

Based on the interim data of many clinical trials, the COVID-19 vaccine was estimated to be effective in preventing severe-to-moderate COVID-19 clinical symptoms.
The efficacy varied across vaccine companies and across trial settings (62-95\%), though recent evidence showed a promising sign that the vaccine might be able to prevent disease transmission. ${ }^{11,12}$ This created a contentious policy discourse and widespread public debate in Thai society about whether the COVID-19 vaccine could be a useful weapon to fight against COVID-19, especially in Samut Sakhon where the epidemic was still active and social distancing was difficult to implement due to the crowded living conditions of migrant workers.

At the time of writing (January 2021), there had been no epidemiological study in Thailand on the effectiveness of COVID-19 vaccines and ACF as the first batch of COVID-19 vaccines was scheduled to arrive in Thailand in the first quarter of 2021. The DDC therefore commissioned a group of researchers in the DOE to estimate if and to what extent the vaccination policies, as well as ACF, could mitigate the outbreak in Samut Sakhon in a timely manner. These policy options included (i) extensive ACF with isolation of positive cases; (ii) vaccination measures to Samut Sakhon residents; and (iii) a combination of ACF and vaccination measures. All of these inform the objective of this study.

\section{Methods}

\section{Study Design}

We applied a secondary analysis on quantitative data. Most model parameters were obtained from the internal database of the DOE and Samut Sakhon Provincial Public Health Office (PPHO). A further review was performed on the MEDLINE database. The review focused on articles published during 2019-2020. As the aim of the review was more to identify key parameters to serve as inputs for the model, rather than answering any specific research questions, no specific inclusion and exclusion criteria were set on the literature search. Some common search terms (such as "COVID-19", "SARS-CoV-2", "generation time" and "serial interval") were utilized. If the interested parameters could not be identified from the recruited literature, we relied on the opinions of epidemiological experts of the MOPH. More details on the parameters are presented later in sub-section, "Model validation and parameter list".

\section{Model Framework}

We used a compartmental susceptible-exposed-infectedrecovered (SEIR) model as the base framework to assess 
the likely impact in a hypothesised population $(\mathrm{N}=$ $10,000)$ if $\mathrm{ACF}$ and vaccination measures are put into effect in Samut Sakhon. The SEIR model categorised the population into four compartments: the susceptible, the exposed (but not infectious), the infected and the recovered. Susceptible people would become infected once having contact with infected cases. ${ }^{13}$ The rate of transferring from a susceptible compartment to an exposed compartment was determined by the reproduction number $\left(\mathrm{R}_{0}\right){ }^{14}$ The incubation period determined the speed of switching from an exposed compartment to an infected compartment. The length of hospital stay governed how fast a patient transferred to a recovered compartment. We divided the population into five categories (asymptomatic, mild, moderate, severe [needing intensive care], and dead). We also incorporated the concept of system dynamics in the base SEIR model to reflect the actual field operation. The infected compartment was split into two compartments, namely, "infected before isolation" and "infected after isolation". We proposed that the benefit of ACF was mainly the reduction of time lag between being infected and reaching isolation by approximately $50 \%$. Since, at the time of writing, the consensus on the efficacy of vaccine from various companies was yet to be finalised, we referred to the recommendation of the World Health Organization (WHO) that a widely deployed COVID-19 vaccine would be effective if the primary efficacy endpoint is at least $50 \%{ }^{15}$ In this regard, we used a figure of $50 \%$ as the vaccine efficacy parameter for transmission reduction. The simplified model framework is elicited in Figure 1.

\section{Model Assumptions and Interested Outcomes}

The model relied on a few key assumptions. Firstly, we assumed that the ACF did not operate all the time but functioned in a biweekly fashion. Secondly, there was inand out-migration to and from the province. Thirdly, it is presumed that mass vaccination for a target population could be performed within a day. Fourthly, a contact between a case and each susceptible person took place at random. Lastly, all infected persons were treated at health facilities. The outcomes of interest were: (i) daily incident cases; (ii) cumulative cases; (iii) cumulative deaths; and (iv) prevalent intensive-care-unit (ICU) bed demand. As, in actual operation, vaccine coverage and degree of ACF intensity might vary. Hence, we analysed nine policy scenarios to aid policy decision, Table 1 .

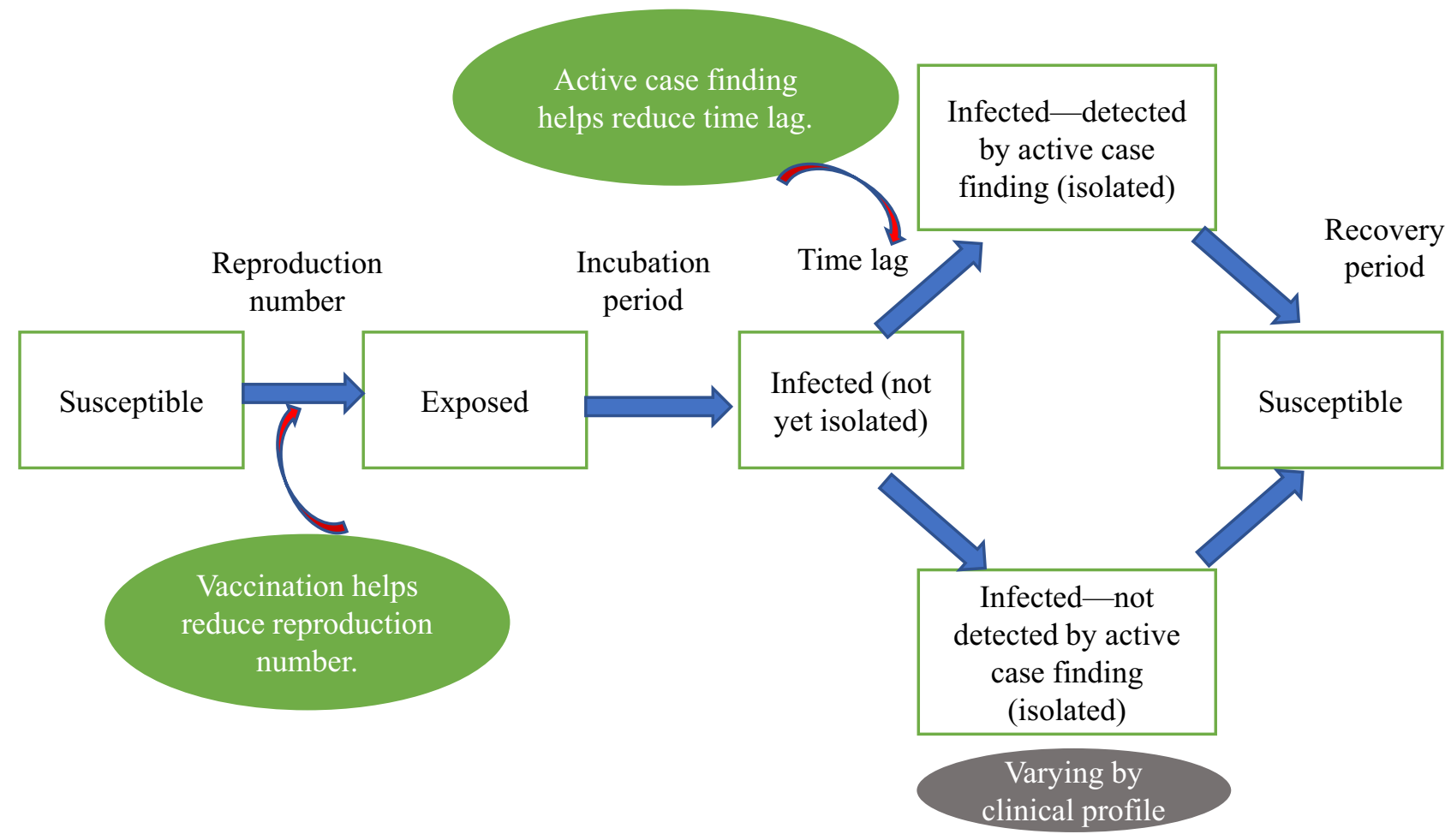

Figure I Simplified model framework. 
Table I Policy Scenarios of Interest

\begin{tabular}{|l|c|c|}
\hline Scenario & $\begin{array}{c}\text { Vaccination } \\
\text { Coverage (\%) }\end{array}$ & $\begin{array}{c}\text { Active Case Finding } \\
\text { Coverage (\%) }\end{array}$ \\
\hline no-VAC \& no-ACF & None & None \\
no-VAC \& ACF50 & None & 50 \\
no-VAC \& ACF90 & None & 90 \\
\hline VAC50 \& no-ACF & 50 & None \\
VAC50 \& ACF50 & 50 & 50 \\
VAC50 \& ACF90 & 50 & 90 \\
\hline VAC90 \& no-ACF & 90 & None \\
VAC90 \& ACF50 & 90 & 50 \\
VAC90 \& ACF90 & 90 & 90 \\
\hline
\end{tabular}

Abbreviations: no-VAC, no vaccination; no-ACF, no active case finding; ACF50, active case finding with $50 \%$ coverage; ACF90 = active case finding with $50 \%$ coverage; VAC50, vaccination with $50 \%$ coverage; VAC 90 , vaccination with $90 \%$ coverage.

\section{Model Validation and Parameter List}

We calibrated $\mathrm{R}_{0}$ by recent evidence on new daily cases in Samut Sakhon between 1 Jan 2021 and 21 Jan 2021. During the peak of outbreak, the effective reproduction number of Samut Sakhon exceeded 3 with a range from 0.2 to $5.6^{8}$ Two meetings among $10-15$ epidemiologists and public health experts in the DDC were held as part of model validation. We found that replacing $R_{0}$ with 3 soundly reflected the actual situation in the province. Stella 2.0 (number: 251-401-786-859) was used to run the model. Tables 2-3 display key parameters and the essential formula of the model.

\section{Sensitivity Analysis}

Sensitivity analysis was performed as complementary to the main analysis. While the main analysis relied on $\mathrm{R}_{0}$ of 3 , this analysed the change in cumulative case volume if $\mathrm{R}_{0}$ changed to 1.5 and 2 . We compared the percentage reduction of cumulative cases in each scenario against "no-VAC \& no-ACF" scenario.

\section{Results}

From a macro perspective, ACF-containing policies (eg, "no-VAC \& ACF90" and "no-VAC \& ACF50") demonstrated more daily incident cases at the very beginning of the outbreak ( $200-250$ cases per day) compared with a "no-VAC \& no-ACF" measure. However, after a week, the "no-VAC \& no-ACF" policy showed an upward trend and reached a peak of about 260 cases per day, by day 25 . The ACF-containing policies displayed a sharp spike of the incident cases by day 30 , followed by a rapid decline in cases. Given the same ACF coverage, the greater the vaccination coverage was, the smaller the spike presented. "VAC90 \& ACF90" policy saw the lowest number of incident cases relative to other policies, Figure 2.

By day 90, the "no-VAC \& ACF90" policy contributed to about 10,500 cases, the largest among all scenarios. "VAC50 \& no-ACF" and "no-VAC \& ACF50" policies came second ( 2900 cases), followed by "VAC-90 \& noACF", "no-VAC \& ACF-90" and "VAC50 \& ACF50" policies ( $\sim 9000-9300$ cases).

If the vaccination covered $90 \%$ of the population in combination with 50\% ACF coverage (VAC90 \& ACF50) or vice versa (VAC50 \& ACF90), the cumulative case toll dropped to approximately 8000-8200. The "VAC90 \& ACF90" policy resulted in the least volume of cases ( $\sim 7000$ cases $)$, Figure 3.

All policies displayed almost the same number of cases during the first two weeks, then demonstrated the largest difference by day 40 , and converged to same level again after day 80. The widest gap of cases needing ICU beds was observed when we compared "no-VAC \& no-ACF" ( $\sim 35$ cases) with "VAC90 \& ACF90" ( 20 cases). The case volume of other scenarios presented somewhere between "no-VAC \& no-ACF" and "VAC90 \& ACF90" policies, Figure 4.

The death toll varied across policy scenarios by a fine margin. "VAC50 \& no-ACF", "no-VAC \& no-ACF", and "no-VAC \& ACF50" policies yielded approximately four cases by the end of the analysis time. "VAC90 \& ACF90" policy exhibited fewer than three deaths in total, the smallest figure when compared with other scenarios, Figure 5.

Sensitivity analysis revealed that vaccination and ACF measures produced the greatest benefit in the lens of percentage reduction in total case volume when $\mathrm{R} 0$ was 2. Given R0 equalling 1.5 or 3, the benefit still presented but with a lesser extent. For instance, with "no-VAC \& noACF" as a reference, "VAC50 \& ACF50" contributed to a $38 \%$-decline in the accumulative case number when R0 amounted to 2, but the corresponding figure appeared to be $-30 \%$ and $-14 \%$ when R0 was 1.5 and 3 respectively, Table 4 .

\section{Discussion}

Overall, this study confirmed that a combination of vaccination and $\mathrm{ACF}$ measures contributed to favourable results in minimising the case volume and death toll. The greater the vaccination and ACF covered, the greater the volume of cases averted. In addition, the benefit of all combined 
Table 2 List of Key Parameters

\begin{tabular}{|c|c|c|c|}
\hline Parameter & Unit & Value & Reference \\
\hline Reproduction number & Dimensionless & 3 & Model calibration \\
\hline Setting population & Persons & 10,000 & $\begin{array}{l}\text { Estimated population size of a migrant populated community in Samut } \\
\text { Sakhon based on experience of the locals }\end{array}$ \\
\hline $\begin{array}{l}\text { Prevalence of infectees at the beginning } \\
\text { of the outbreak (\%) }\end{array}$ & Dimensionless & 10 & Expert opinions \\
\hline Average incubation period & Days & 5.2 & Li et al $^{16}$ \\
\hline $\begin{array}{l}\text { Infectious duration and gap between } \\
\text { infected and isolated }\end{array}$ & Days & 7 & Byrne et $\mathrm{al}^{32}$ and expert opinions \\
\hline Vaccine efficacy (\%) & Dimensionless & 50 & World Health Organization ${ }^{15}$ \\
\hline Clinical profile: asymptomatic (\%) & Dimensionless & 56.6 & Internal database of the Department of Disease Control \\
\hline Clinical profile: mild (\%) & Dimensionless & 42.2 & Internal database of the Department of Disease Control \\
\hline Clinical profile: moderate (\%) & Dimensionless & 0.9 & Internal database of the Department of Disease Control \\
\hline Clinical profile: severe (\%) & Dimensionless & 0.2 & Internal database of the Department of Disease Control \\
\hline Clinical profile: dead (\%) & Dimensionless & 0.1 & Internal database of the Department of Disease Control \\
\hline Hospitalisation days: asymptomatic & Days & 10 & Internal database of the Department of Disease Control \\
\hline Hospitalisation days: mild & Days & 10 & Internal database of the Department of Disease Control \\
\hline Hospitalisation days: moderate & Days & 14 & Internal database of the Department of Disease Control \\
\hline Hospitalisation days: severe & Days & 30 & Internal database of the Department of Disease Control \\
\hline Hospitalisation days: death & Days & 30 & Internal database of the Department of Disease Control \\
\hline Time horizon for the analysis & Days & 90 & Expert opinions \\
\hline
\end{tabular}

Table 3 Essential Formula of the Model

\begin{tabular}{|c|c|c|}
\hline Change of Status & Formula 33,34 & Note \\
\hline From susceptible to exposed & $\begin{array}{l}-\left(\mathrm{R}_{0} / \mathrm{Dinf}\right) *(\mathrm{I}-\mathrm{VE}) \\
* \mathrm{VC} * \mathrm{~S} * \mathrm{lb} / \mathrm{P}\end{array}$ & $\begin{array}{l}\text { Dinf = infectious duration, } \mathrm{lb}=\text { Infectees (before isolation), } \mathrm{P}=\text { total } \\
\text { population, } \mathrm{R}_{0}=\text { basic reproduction number, } \mathrm{S}=\text { susceptible population, } \\
\mathrm{VC}=\text { vaccination coverage, } \mathrm{VE}=\text { vaccine efficacy }\end{array}$ \\
\hline $\begin{array}{l}\text { From susceptible to infected (before } \\
\text { isolation) }\end{array}$ & -E/Dinc & Dinc $=$ incubation period, $E=$ Exposed population \\
\hline $\begin{array}{l}\text { From infected (before isolation) to infected } \\
\text { (after isolation) from active case finding }\end{array}$ & $\begin{array}{l}-\mathrm{ACFC} *(\mathrm{lb} /(\mathrm{Dlag} * \\
(\mathrm{I}-\mathrm{ACFE}))\end{array}$ & $\begin{array}{l}\text { ACFC = active case finding coverage, } A C F E=\text { active case finding } \\
\text { effectiveness, Dlag = lag days from infected to isolation, } l b=\text { Infectees } \\
\text { (before isolation) }\end{array}$ \\
\hline $\begin{array}{l}\text { From infected (before isolation) to infected } \\
\text { (after isolation) from routine health services }\end{array}$ & $-(\mathrm{I}-\mathrm{ACFC})^{*}(\mathrm{Ib} /(\mathrm{Dlag} *)$ & $\begin{array}{l}\mathrm{ACFC}=\text { active case finding coverage, } \mathrm{Dlag}=\text { lag days from infected to } \\
\text { isolation, } \mathrm{lb}=\text { Infectees (before isolation) }\end{array}$ \\
\hline From infected to recovered & $-l a / D r x$ & Drx $=$ hospitalisation days, la $=$ Infectees (after isolation) \\
\hline
\end{tabular}

Copyright (C) Springer Nature B.V. 2020. 


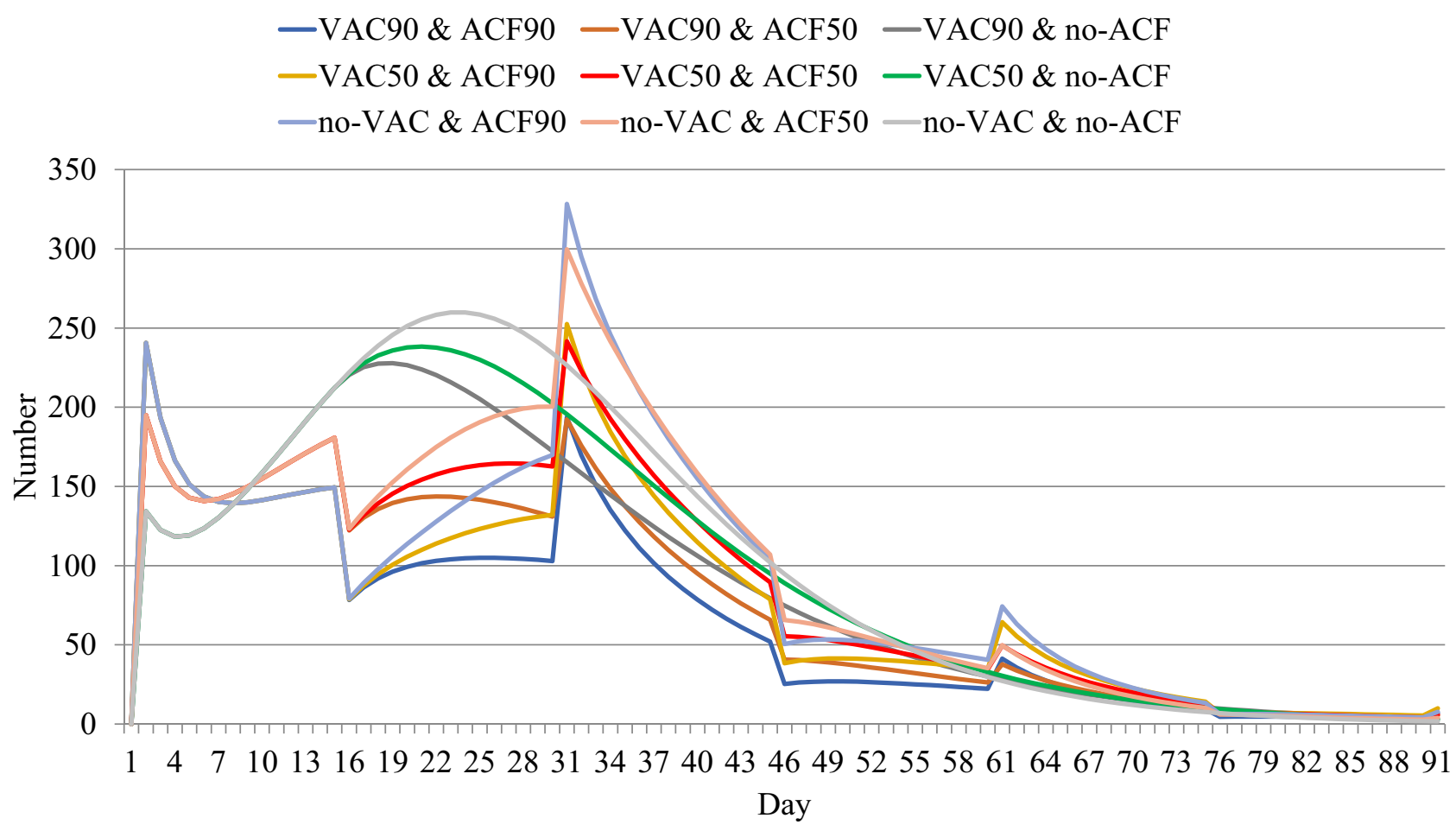

Figure 2 Daily incident cases by policy scenarios.

Abbreviations: no-VAC, no vaccination; no-ACF, no active case finding; ACF50, active case finding with $50 \%$ coverage; ACF 90 , active case finding with $50 \%$ coverage; VAC50, vaccination with $50 \%$ coverage; VAC 90 , vaccination with $90 \%$ coverage.

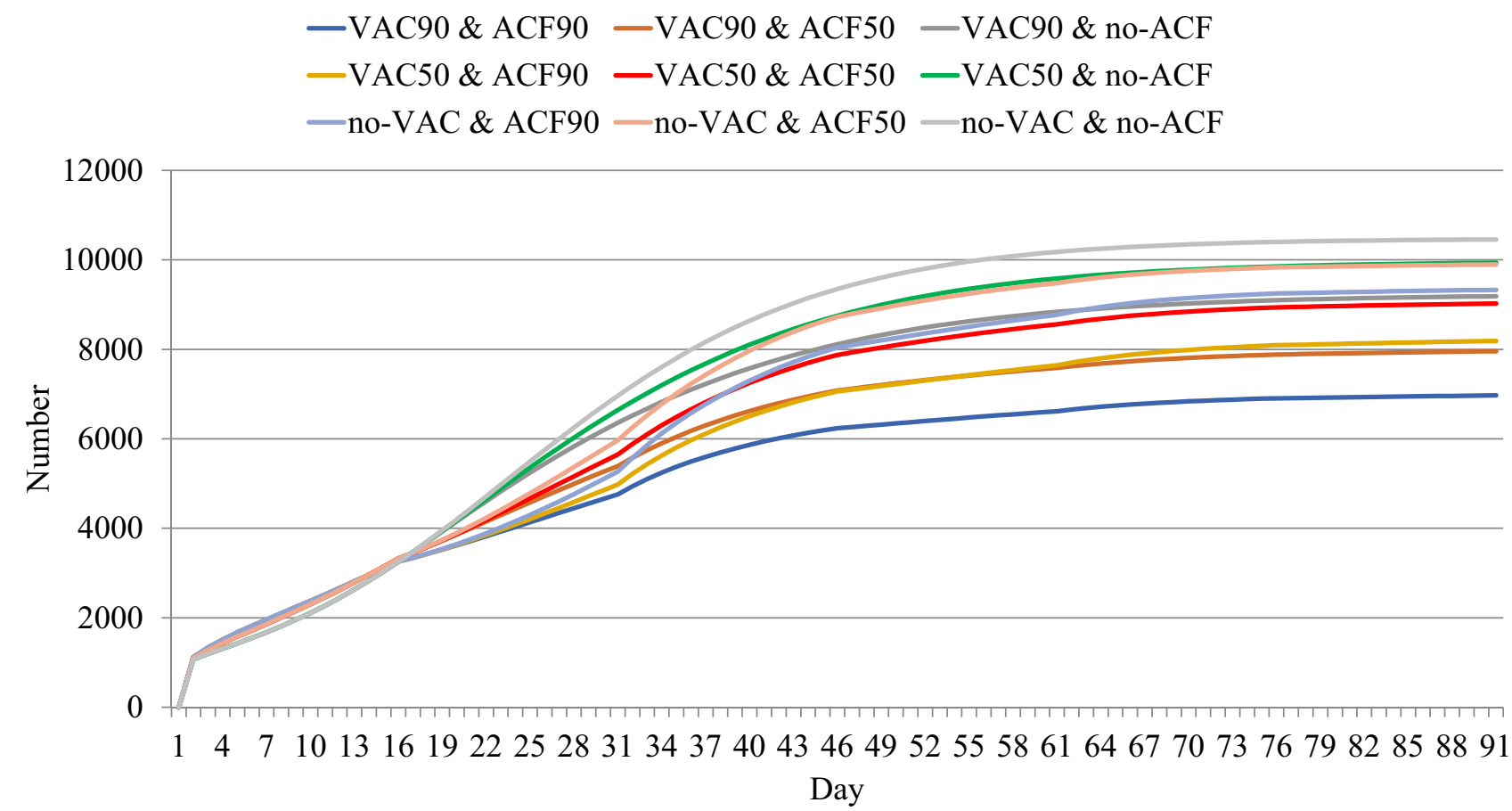

Figure 3 Cumulative cases by policy scenarios.

Abbreviations: no-VAC, no vaccination; no-ACF, no active case finding; ACF50, active case finding with $50 \%$ coverage; ACF90, active case finding with $50 \%$ coverage; VAC50, vaccination with $50 \%$ coverage; VAC 90 , vaccination with $90 \%$ coverage. 


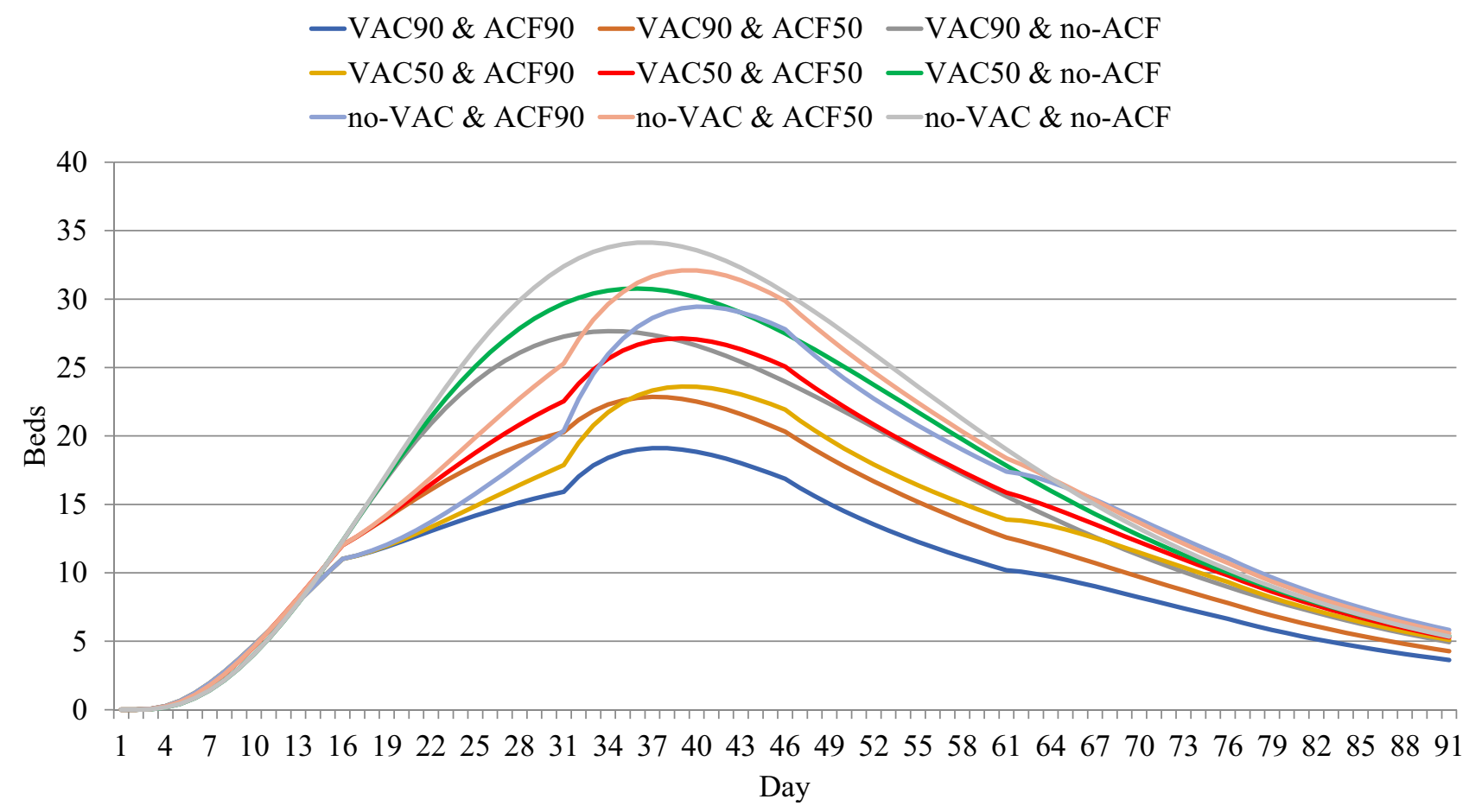

Figure 4 Prevalent cases needing intensive care beds.

Abbreviations: no-VAC, no vaccination; no-ACF, no active case finding; ACF50, active case finding with $50 \%$ coverage; ACF 90 , active case finding with $50 \%$ coverage; VAC50, vaccination with $50 \%$ coverage; VAC 90 , vaccination with $90 \%$ coverage.

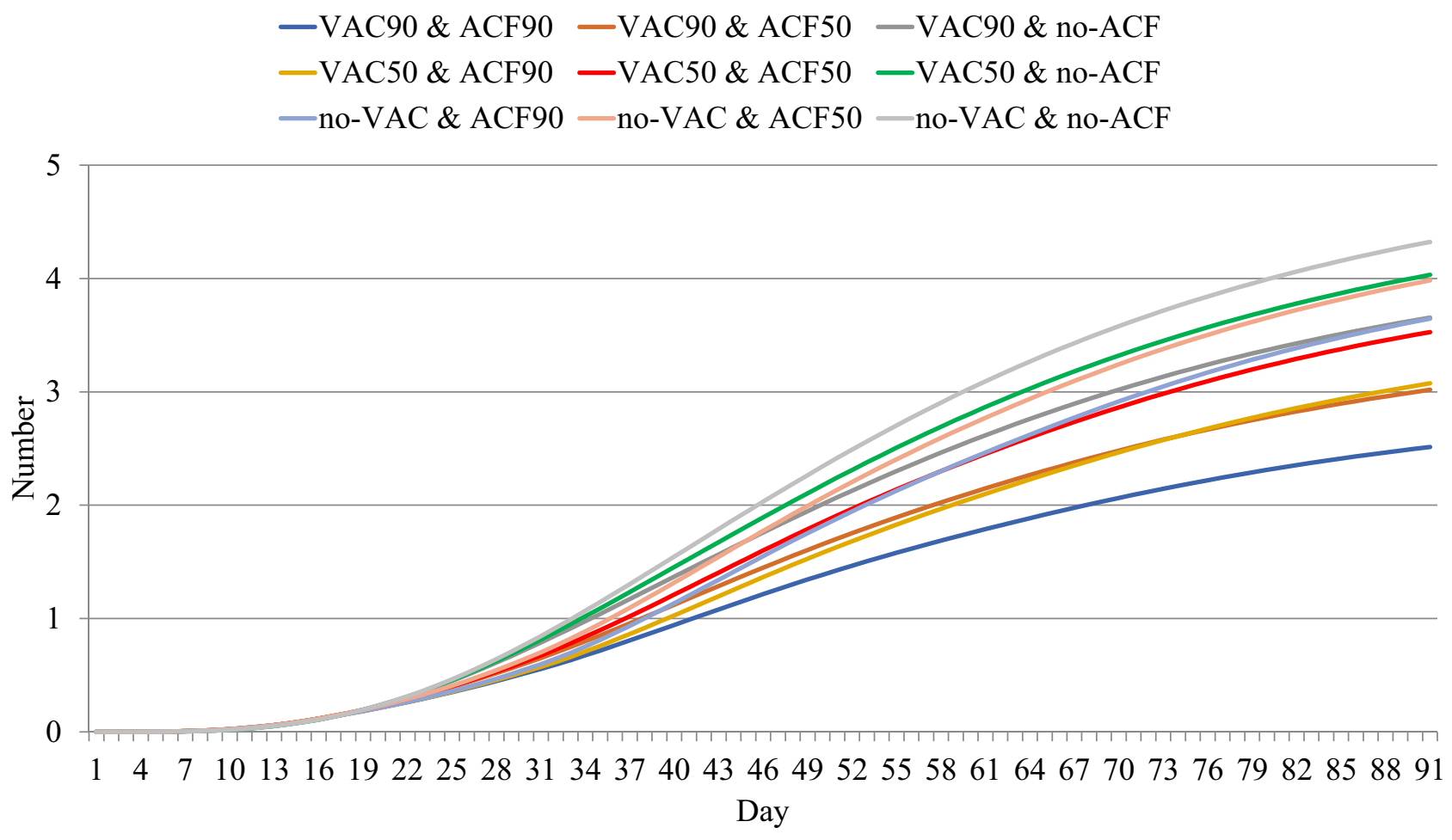

Figure 5 Cumulative deaths by policy scenarios.

Abbreviations: no-VAC, no vaccination; no-ACF, no active case finding; ACF50, active case finding with $50 \%$ coverage; ACF90, active case finding with $50 \%$ coverage; VAC50, vaccination with $50 \%$ coverage; VAC 90 , vaccination with $90 \%$ coverage. 
Table 4 Reduction of Cumulative Cases by Day 90 Between Each Policy and "No-VAC \& No-ACF” Policy

\begin{tabular}{|l|c|c|c|c|c|c|}
\hline & \multicolumn{3}{|c|}{ Volume } & \multicolumn{3}{c|}{ Percent Reduction } \\
\cline { 2 - 7 } & $\mathbf{R}_{\mathbf{0}}=1.5$ & $\mathbf{R}_{\mathbf{0}}=\mathbf{2}$ & $\mathbf{R}_{\mathbf{0}}=\mathbf{3}$ & $\mathbf{R}_{\mathbf{0}}=1.5$ & $\mathbf{R}_{\mathbf{0}}=\mathbf{2}$ & $\mathbf{R}_{\mathbf{0}}=\mathbf{3}$ \\
\hline no-VAC \& no-ACF & 9132 & 7337 & 10,457 & Reference & Reference & Reference \\
no-VAC \& ACF50 & 7755 & 5579 & 9895 & $-15 \%$ & $-24 \%$ & $-5 \%$ \\
no-VAC \& ACF90 & 6616 & 4525 & 9330 & $-28 \%$ & $-38 \%$ & $-11 \%$ \\
VAC50 \& no-ACF & 7963 & 6022 & 9935 & $-13 \%$ & $-18 \%$ & $-5 \%$ \\
VAC50 \& ACF50 & 6363 & 4556 & 9024 & $-30 \%$ & $-38 \%$ & $-14 \%$ \\
VAC50 \& ACF90 & 5290 & 3812 & 8188 & $-42 \%$ & $-48 \%$ & $-22 \%$ \\
VAC90 \& no-ACF & 6793 & 5084 & 9187 & $-26 \%$ & $-31 \%$ & $-12 \%$ \\
VAC90 \& ACF50 & 5318 & 3980 & 7960 & $-42 \%$ & $-46 \%$ & $-24 \%$ \\
VAC90 \& ACF90 & 4473 & 3446 & 6971 & $-51 \%$ & $-53 \%$ & $-33 \%$ \\
\hline
\end{tabular}

Abbreviations: no-VAC, no vaccination; no-ACF, no active case finding; ACF50, active case finding with $50 \%$ coverage; ACF90, active case finding with $50 \%$ coverage; VAC50, vaccination with $50 \%$ coverage; VAC 90 , vaccination with $90 \%$ coverage.

strategies in terms of total case reduction would be maximised if the epidemic activity, as reflected by $\mathrm{R}_{0}$, was not too intense.

This finding corroborated the ideas of many previous studies that ACF is a key measure to contain and suppress the epidemic. ${ }^{16}$ For example, China reported the benefit of ACF to identify patients in epidemic communities. ${ }^{16}$ The ACF in China was conducted not only by the state but also by the network of communities. Examples of countries that were also successful in containing the outbreak through the use of ACF were Mongolia, Singapore, South Korea, and Vietnam. ${ }^{17-19}$ Singapore used a proactive strategy to detect the suspected patients through a public prevention clinic network and promulgated the home quarantine orders for patients with mild illnesses.20,21 South Korea greatly expanded the screening sites for SARS-CoV-2 nucleic acid tests to encompass asymptomatic cases as many as possible. This included the use of public health-care clinics, drive-through centres, and walkin screening sites. ${ }^{22,23}$

Traoré and Konané suggested that the contact tracing strategy, as well as ACF, can reduce $R_{0}$ to values below unity as intended for disease control, but effective control of the epidemic can be achieved when the effectiveness of contact tracing is high, and $\mathrm{R}_{0}$ is not too large. In the population where $\mathrm{R}_{0}$ is large, the epidemic may not be controlled using an ACF strategy alone. ${ }^{24}$ Our findings also upheld the idea that such a vaccination policy hugely complements the ACF measure. The situation in Samut Sakhon is very complex because the city is extremely urbanised and migrant residents are mostly living in densely populated conditions. These conditions create remarkable difficulties for ACF and other non-pharmaceutical interventions (NPI), such as physical distancing measures and individual risk modifications. At present, ACF is the major intervention in Samut Sakhon with an aim to test all 400,000 workers and isolate those who are positive for 10 days in field hospitals or factory dormitories. So far, the Government has built approximately 3000 field hospital beds. Healthcare providers use individual nasopharyngeal swabs for real-time polymerase chain reaction (Rt-PCR) testing. By average it takes at least 48 hours to obtain the swab result. This means ACF alone may not be able to detect and isolate cases as early as expected. Therefore, the Thai Government should consider an urgent launch of a vaccination policy in Samut Sakhon or in any similar settings once the COVID-19 vaccines are available.

The bottom line is, at the time of writing, the evidence of vaccine effectiveness against COVID-19 transmission is not yet fully understood. ${ }^{25}$ Many different endpoints are used in vaccine research to define efficacy depending on the pathogen, consequences of infection, and transmission dynamics. Outcomes of most randomised controlled trials (RCT) are presented as a proportional decline in disease between vaccinated participants and control participants. ${ }^{26}$ Other outcomes might include assessing sterilising immunity, severity of resultant clinical disease, and duration of infectivity. Besides, RCTs almost always represent bestcase scenarios of vaccine efficacy under idealised conditions; but, in the real world, vaccine efficacy does not always predict vaccine effectiveness and such effectiveness is likely to vary across age groups and people from different walks of life as certain subpopulations in society may always face greater risks of infection or may be more 
vulnerable than others. ${ }^{27}$ However, the findings above are of certain value for policy consideration as the vaccine efficacy parameter applied in the model was very modest (only 50\%) while recent evidence demonstrated much more favourable outcomes than the $50 \%$ figure. ${ }^{12}$ For instance, the latest interim analysis from a Phase 3 clinical trial in Russia by Logynov et al demonstrated that an rAd26 and rAd5 vector-based heterologous prime-boost COVID-19 vaccine (Sputnik V) showed 91.6\% efficacy against COVID-19 and was well tolerated in a large cohort. ${ }^{11,28,29}$

Caution should be exercised when interpreting our findings as different models almost always rely on different assumptions, structures and parameters (even if they explored a situation in the same setting). For instance, the SEIR model conducted by the International Health Policy Programme in late December 2020 predicted that the daily incident cases of Samut Sakhon would number up to 2700 by early February 2021 given the overall effectiveness of measures (including ACF, vaccines and other social measures combined). ${ }^{30}$ This figure was approximately eightfold larger than our estimates. However, this is not surprising as the model postulated that the value of $\mathrm{R}_{0}$ equalled 4 - to reflect the force of infection at the beginning of the outbreak in Samut Sakhon - while our study used a much smaller $R_{0}$, as when we initiated the study the magnitude of outbreak had already subsided to certain degree (since some measures were already introduced). ${ }^{30}$ Hence, the most important value of the modelling study was to provide a clearer insight for policy decision-making for resource planning rather than identifying a perfect accuracy for forecasted numbers. ${ }^{31}$

Some limitations remain in this study. Firstly, most of the parameters applied in the model derived from the epidemic situation in Samut Sakhon. Therefore, a generalisation of the findings to other areas should be made with caution; though one may use the approach in this study as an analysis example in any similar settings. Secondly, during the period of epidemic, it is almost always difficult to conduct primary research to obtain empirical evidence as the utmost priority of the field operations was to curb the epidemic. Accordingly, many parameters in the model were obtained through authors' assumptions. Though we tried to validate the findings against the opinions of experts and local providers, this does not substitute the use of empirical data. Thirdly, the model applied a deterministic approach as it is more convenient to communicate with policy makers, compared with a stochastic approach and because most parameters in the model lacked information of the distribution characteristics, which is a prerequisite for stochastic analysis. Last but not least, though we demonstrated the benefit of vaccination strategies in this setting, in real practice, actual implementation should consider many more policy angles; for instance, social acceptability (if migrants are to be the vaccination target before Thai citizens), cost-effectiveness of the policies, and operational feasibility. Further studies that address these topics are of great value. In addition, a close monitoring of the information in the field is useful, not only for the benefit of disease control but also for obtaining empirical evidence which will help refine and validate the model.

\section{Conclusion}

This study reaffirmed the idea that a combination of vaccination and ACF measures contributed to favourable results in reducing the number of COVID-19 cases and deaths, relative to the implementation of a single measure. The greater the vaccination and ACF coverage, the greater the volume of cases saved. We also discovered that over a three-month period of operating vaccination and ACF measures with $90 \%$ coverage, the case toll would drop by $33 \%$ compared with the scenario where no measures were implemented. Additionally, the value of all combined strategies in terms of total case reduction would be maximised if the epidemic activity as reflected by $\mathrm{R}_{0}$ was not too pronounced. To operationalise the vaccination policy in combination with ACF measures, policy makers should consider the readiness of health resources and the issue of social acceptability since COVID-vaccines are urgently needed by not only migrants but also all populations in the target area. Therefore, further studies that aim to explore the policy feasibility as well as the prioritisation of COVID-19 vaccines and other health resources are recommended. Additional research that uses empirical evidence should be conducted to complement our study that employed the analysis on secondary data, and this would help provide useful information to monitor the effectiveness of public health measures in the field.

\section{Abbreviations}

ACF, active case finding; COVID-19, Coronavirus Disease 2019; DDC, Department of Disease Control; DOE, Division of Epidemiology; MOPH, Ministry of Public 
Health; NPI, Non-pharmaceutical interventions; PPHO, Provincial Public Health Office; RCT, Randomised controlled trials; Rt-PCR, Real-time polymerase-chain reaction; SEIR, susceptible-exposed-infected-recovered; VAC, vaccination; WHO, World Health Organization.

\section{Data Sharing Statement}

The datasets generated and/or analysed during the current study are not publicly available due to the DDC's regulation but are available from the corresponding author on reasonable request.

\section{Ethics Approval and Consent to Participate}

The study did not involve human participation, excepting the process of seeking opinions' experts on model validity. Almost all the analysis was performed via secondary data. This study obtained ethics approval from the Institute for the Development of Human Research Protections (IHRP), letter head IHRP 985/2563.

\section{Acknowledgments}

The investigators are immensely grateful for the support from the DDC's and the IHPP's staff. Comments and advice from all faculties of the Field Epidemiology Training Program (FETP) of the DOE are hugely appreciated.

\section{Author Contributions}

All authors made a significant contribution to the work reported, whether that is in the conception, study design, execution, acquisition of data, analysis and interpretation, or in all these areas; took part in drafting, revising or critically reviewing the article; gave final approval of the version to be published; have agreed on the journal to which the article has been submitted; and agreed to be accountable for all aspects of the work.

\section{Funding}

This study received funding support from Health Systems Research Institute [Contract No. 63-162].

\section{Disclosure}

The authors declare that they have no conflicts of interest for this work.

\section{References}

1. Huang C, Wang Y, Li X, et al. Clinical features of patients infected with 2019 novel coronavirus in Wuhan, China. Lancet. 2020;395 (10223):497-506. doi:10.1016/S0140-6736(20)30183-5

2. Jee Y. WHO international health regulations emergency committee for the COVID-19 outbreak. Epidemiol Health. 2020;42:e2020013. doi:10.4178/epih.e2020013

3. World Health Organization. Listings of WHO's response to COVID19. Geneva: WHO; 2020 [cited February 15, 2021]. Available from: https://www.who.int/news/item/29-06-2020-covidtimeline. Accessed July 21, 2021.

4. Johns Hopkins Coronavirus Resource Center. Daily confirmed new cases. Baltimore, Maryland: Johns Hopkins University School of Medicine; 2021 [cited February 15, 2021]. Available from: https:// coronavirus.jhu.edu/data/new-cases]. Accessed July 21, 2021.

5. Doung-Ngern P, Suphanchaimat R, Panjangampatthana A, et al. Case-control study of use of personal protective measures and risk for SARS-CoV 2 infection, Thailand. Emerg Infect Dis. 2020;26 (11):2607-2616. doi:10.3201/eid2611.203003

6. Namwat C, Suphanchaimat R, Nittayasoot N, Iamsirithaworn S. Thailand's response against coronavirus disease 2019: challenges and lessons learned. OSIR. 2020;13(1):33-37.

7. WHO Thailand Country Office. Coronavirus disease 2019: situation report. Nonthaburi: WHO; 2021 [cited February 15, 2021]. Available from: https://cdn.who.int/media/docs/default-source/searo/thailand/ 2021_1_25_eng_sitrep_135-covid19.pdf?sfvrsn=a034c3d5_3. Accessed July $21,202 \overline{1}$.

8. Department of Disease Control. Coronavirus Disease (COVID-19): Thailand Situation. Nonthaburi: Department of Disease Control, Ministry of Public Health; 2021 [cited February 15, 2021]. Available from: https://ddc.moph.go.th/viralpneumonia/eng/index. php. Accessed July 21, 2021.

9. Luksamijarulkul P, Suknongbung S, Vatanasomboon P, Sujirarut D. Health status, environmental living conditions and microbial indoor air quality among migrant worker households in Thailand. Southeast Asian J Trop Med Public Health. 2017;48(2):396-406.

10. Thanthong-Knight $R$. Thailand tightens measures to stem new wave of infections. New York: Bloomberg; 2020. Available from: https:// www.bloomberg.com/news/articles/2020-12-29/virus-hit-thailandtightens-curbs-to-stem-new-wave-of-infections. Accessed July 21, 2021.

11. Polack FP, Thomas SJ, Kitchin N, et al. Safety and efficacy of the BNT162b2 mRNA covid-19 vaccine. $N$ Engl J Med. 2020;383 (27):2603-2615. doi:10.1056/NEJMoa2034577

12. Knoll MD, Wonodi C. Oxford-AstraZeneca COVID-19 vaccine efficacy. Lancet. 2021;397(10269):72-74. doi:10.1016/S0140-6736(20) 32623-4

13. Brauer F. Compartmental models in epidemiology. Math Epidemiol. 2008;1945:19-79.

14. Dietz K. The estimation of the basic reproduction number for infectious diseases. Stat Methods Med Res. 1993;2(1):23-41. doi:10.1177/ 096228029300200103

15. World Health Organization. Considerations for evaluation of COVID19 vaccines: points to consider for manufacturers of COVID19 vaccines. WHO: Geneva; 2020 [cited February 16, 2021]. Available from: https://www.who.int/medicines/regulation/pre qualification/prequal-vaccines/WHO_Evaluation_Covid_Vaccine. pdf?ua=1. Accessed July 21, 2021.

16. Li Z, Chen Q, Feng L, et al. Active case finding with case management: the key to tackling the COVID-19 pandemic. Lancet. 2020;396 (10243):63-70. doi:10.1016/S0140-6736(20)31278-2

17. Kuguyo O, Kengne AP, Dandara C. Singapore COVID-19 pandemic response as a successful model framework for low-resource health care settings in Africa? OMICS. 2020;24(8):470-478. doi:10.1089/ omi.2020.0077 
18. Tabari P, Amini M, Moghadami M, Moosavi M. International public health responses to COVID-19 outbreak: a rapid review. Iran J Med Sci. 2020;45(3):157-169.

19. Yamamoto N, Bauer G. Apparent difference in fatalities between Central Europe and East Asia due to SARS-COV-2 and COVID-19: four hypotheses for possible explanation. Med Hypotheses. 2020;144:110160. doi:10.1016/j.mehy.2020.110160

20. Lai SHS, Tang CQY, Kurup A, Thevendran G. The experience of contact tracing in Singapore in the control of COVID-19: highlighting the use of digital technology. Int Orthop. 2021;45(1):65-69. doi:10.1007/s00264-020-04646-2

21. Chen JI, Yap JC, Hsu LY, Teo YY. COVID-19 and Singapore: from early response to circuit breaker. Ann Acad Med Singapore. 2020;49 (8):561-572. doi:10.47102/annals-acadmedsg.2020239

22. Choi S, Han C, Lee J, Kim SI, Kim IB. Innovative screening tests for COVID-19 in South Korea. Clin Exp Emerg Med. 2020;7(2):73-77. doi: $10.15441 /$ ceem. 20.032

23. Kang J, Jang YY, Kim J, et al. South Korea's responses to stop the COVID-19 pandemic. Am J Infect Control. 2020;48(9):1080-1086. doi:10.1016/j.ajic.2020.06.003

24. Traoré A, Konané FV. Modeling the effects of contact tracing on COVID-19 transmission. Adv Differ Equ. 2020;2020(1):509. doi:10.1186/s13662-020-02972-8

25. Hodgson SH, Mansatta K, Mallett G, Harris V, Emary KRW, Pollard AJ. What defines an efficacious COVID-19 vaccine? A review of the challenges assessing the clinical efficacy of vaccines against SARSCoV-2. Lancet Infect Dis. 2021;21(2):e26-e35. doi:10.1016/S14733099(20)30773-8

26. Weinberg GA, Szilagyi PG. Vaccine epidemiology: efficacy, effectiveness, and the translational research roadmap. $J$ Infect Dis. 2010;201(11):1607-1610. doi:10.1086/652404
27. Hanquet G, Valenciano M, Simondon F, Moren A. Vaccine effects and impact of vaccination programmes in post-licensure studies. Vaccine. 2013;31(48):5634-5642. doi:10.1016/j.vaccine.2013.07.006

28. Logunov DY, Dolzhikova IV, Shcheblyakov DV, et al. Safety and efficacy of an rAd26 and rAd5 vector-based heterologous primeboost COVID-19 vaccine: an interim analysis of a randomised controlled phase 3 trial in Russia. Lancet. 2021;397(10275):671-681. doi:10.1016/S0140-6736(21)00234-8

29. Baden LR, El Sahly HM, Essink B, et al. Efficacy and Safety of the mRNA-1273 SARS-CoV-2 Vaccine. $N$ Engl J Med. 2021;384 (5):403-416. doi:10.1056/NEJMoa2035389

30. International Health Policy Programme. Rapid forecasting COVID19 situation in Samut Sakorn by math modeling (December 2020 till March 2021). Nonthaburi: IHPP, DDC; 2020.

31. Suphanchaimat R, Thammawijaya P, Wongsanuphat S, Ploddi K. Roles and challenges of mathematical modellings in the era of Coronavirus disease 2019 (COVID-19) pandemic. JHSAM. 2021;3 (1):6-8.

32. Byrne AW, McEvoy D, Collins AB, et al. Inferred duration of infectious period of SARS-CoV-2: rapid scoping review and analysis of available evidence for asymptomatic and symptomatic COVID-19 cases. BMJ Open. 2020;10(8):e039856. doi:10.1136/bmjopen-2020039856

33. Mwalili S, Kimathi M, Ojiambo V, Gathungu D, Mbogo R. SEIR model for COVID-19 dynamics incorporating the environment and social distancing. BMC Res Notes. 2020;13(1):352. doi:10.1186/ s13104-020-05192-1

34. He S, Peng Y, Sun K. SEIR modeling of the COVID-19 and its dynamics. Nonlinear Dyn. 2020;101(3):1-14.
Risk Management and Healthcare Policy

\section{Publish your work in this journal}

Risk Management and Healthcare Policy is an international, peerreviewed, open access journal focusing on all aspects of public health, policy, and preventative measures to promote good health and improve morbidity and mortality in the population. The journal welcomes submitted papers covering original research, basic science, clinical \& epidemiological studies, reviews and evaluations, guidelines, expert opinion and commentary, case reports and extended reports. The manuscript management system is completely online and includes a very quick and fair peer-review system, which is all easy to use. Visit http://www.dovepress.com/testimonials.php to read real quotes from published authors. 